\title{
Gangrenous Cholecystitis with Atypical Presentation in an Elderly Diabetic Woman
}

\author{
Vera Clérigo*, Cláudia Rocha, André Rodrigues, Lígia Fernandes, Dora Sargento, \\ Glória Silva \\ Serviço de Medicina III, Hospital Pulido Valente, Centro Hospitalar Lisboa Norte, Lisboa, Portugal \\ Email: ${ }^{*}$ vclerigo21@gmail.com
}

Received 27 July 2014; revised 20 August 2014; accepted 2 September 2014

Copyright (C) 2014 by authors and Scientific Research Publishing Inc.

This work is licensed under the Creative Commons Attribution International License (CC BY).

http://creativecommons.org/licenses/by/4.0/

(c) (i) Open Access

\begin{abstract}
In elderly patients, numerous factors subsidize the diagnostic challenge and high incidence of complications in this specific population, taking gangrenous cholecystitis as a critical example. The aim of this work is to report an unusual case of gangrenous choleystitis in an elderly diabetic women and its atypical clinical presentation. A 79-year-old female patient came to our observation; her medical history showed nausea and vomiting of about 2 hours which rapidly ended with symptomatic therapy, without recurrence, and a 3-week history of intermittent fever associated with productive cough. No abdominal discomfort was declared. Physical examination of the abdomen was negative. Laboratory analysis revealed leukocytosis with the remaining criteria within the normal range. After 2 days, she started with a mildabdominal pain in the epigastric region that rapidly progressed to the right upper quadrant, right flank and right iliac fossa, without nausea, vomiting or fever. Abdominal computer tomography findings revealed thickness of the gall-bladder and important densification of the vascular bed. Acute cholecystitis was diagnosed. The patient was then submitted to a laparoscopic cholecystectomy under general anaesthesia with findings suggestive of gangrenous acute cholecystitis confirmed by histologic examination of the specimen. Delays in diagnosing acute cholecystitis in specific populations, such as elderly diabetics, result in a higher prevalence of morbidity and mortality due to potentially serious complications as gangrenous cholecystitis. Consequently, the diagnosis should be measured and investigated promptly in order to prevent poor outcomes.
\end{abstract}

\section{Keywords}

Diabetes Mellitus, Elderly, Gangrenous Acute Cholecystitis

\footnotetext{
*Corresponding author.
}

How to cite this paper: Clérigo, V., Rocha, C., Rodrigues, A., Fernandes, L., Sargento, D. and Silva, G. (2014) Gangrenous Cholecystitis with Atypical Presentation in an Elderly Diabetic Woman. Case Reports in Clinical Medicine, 3, 503-507. 


\section{Introduction}

Gangrenous cholecystitis (GC) is considered as a severe complication and contemplates a minority of all patients presenting with acute cholecystitis [1]-[3]. The pathophysiological mechanism includes a gallbladder wall tension associated to intense inflammatory reaction resulting in local or global gallbladder wall ischemia. Vascular compromise secondary to epithelial injury due to sustained obstruction of the cystic duct is also a critical mechanism. Additionally, due to epithelial injury, phospholipases are released and degrade adjacent cell membranes generating an important inflammatory reaction [4] [5]. Progression to gangrenous cholecystitis may take days to hours [6]-[8]. The risk factors for this condition and its influence on morbidity and mortality paralleled with those of non-gangrenous acute cholecystitis (NGAC) are not consistently defined in several studies. It commonly occurs in older and diabetic patients [1] however the pre-operative diagnosis of this condition may denote a clinical challenge. Since the elderly population, especially in developed countries, is growing quickly, the increase of misdiagnosis and overall mortality is a progressively common problem.

The aim of this work is to report on a gangrenous cholecysitis in an elderly diabetic woman, causing a very atypical clinical picture and draw attention to the problematic diagnoses in elderly diabetic populations in particular.

\section{Case Report}

A 79-year-old caucasian woman presented to the emergency department with a two hour history of nausea, vomiting and malaise, which rapidly ended with symptomatic therapy instituted without recurrence. On presentation she had fever and the remaining vital signs were within the normal range. In detail, the patient reported that over the previous three weeks she had had intermittent fever up to $39^{\circ} \mathrm{C}$ associated with productive cough with purulent sputum and started empiric antibiotic therapy with oral amoxicillin plus clavulanic acid (875/125 mg thrice daily) during 24 hours, without significant clinical improvement. These symptoms were not associated with any other abdominal or thoracic signs. Her medical history included essential hypertension, type 2 diabetes mellitus and post-intervention status of breast cancer four years earlier, currently under surveillance. Physical examination of the abdomen was negative. Laboratory blood samples obtained when the patient was febrile, showed several abnormal findings, including leukocytosis $(15,470$ cells/ $\mu \mathrm{L}$; reference range [RR], 4000 - 11,000 cells $/ \mu \mathrm{L}$ ) with neutrophilia (90.8\%) and elevated C-reactive protein $(5.1 \mathrm{mg} / \mathrm{dL} ; \mathrm{RR},<0.5 \mathrm{mg} / \mathrm{dL})$. However, aspartate aminotransferase (13 U/L; RR, <34U/L) and alanine aminotransferase (12 U/L; RR, 12 - $78 \mathrm{U} / \mathrm{L}$ ) levels, serum bilirubin $(0.7 \mathrm{mg} / \mathrm{dL}$; RR, $<1.5 \mathrm{mg} / \mathrm{dL})$, alkaline phosphatase (100 U/L; RR, 45 - $129 \mathrm{U} / \mathrm{L})$ levels and lipase (17 U/L; RR, 13 - $60 \mathrm{U} / \mathrm{L})$ assays were in the normal range. Amylase (3 U/L; RR, 13 - $53 \mathrm{U} / \mathrm{L})$ was diminished. Gammaglutamyl transpeptidase (50 U/L; RR, <38 U/L) was discreetly elevated. Cardiac enzymology and kidney function were normal. Fasting plasma glucose level (152 mg/dL; RR, $70-100 \mathrm{mg} / \mathrm{dL}$ ) and hemoglobin A1C level (6.2\%; RR, 4.0\% - 6.0\%) were elevated. Urinalysis was normal. Electrocardiogram and thoracic radiography were innocent. She was admitted to our hospital with the diagnosis of acute tracheobronchitis and for surveillance. Aerobic and anaerobic blood cultures were negative. The fever disappeared one day after an empiric antibiotic therapy with intravenous amoxicillin plus clavulanic acid (2.2 g thrice daily) and azithromycin (500 mg once daily) was started.

After two days, she started with a mild abdominal pain in the epigastric region that rapidly progressed to the right upper quadrant, right flank and right iliac fossa, without nausea, vomiting or fever. Physical examination showed an abdomen with generalized rebound tenderness and no palpable tender mass in the upper right quadrant with negative Murphy's sign. Laboratory blood tests revealed no leukocytosis $(7.110$ cells/ $\mu \mathrm{L} ; \mathrm{RR}, 4000$ $11,000$ cells $/ \mu \mathrm{L})$ with relative neutrophilia $(90.8 \%)$ and increased C-reactive protein $(34.3 \mathrm{mg} / \mathrm{dL}$; RR, $<0.5$ $\mathrm{mg} / \mathrm{dL}$ ). The remaining laboratory analysis performed, including the previously referred, continued within the normal range with merely gammaglutamyl transpeptidase elevated. Due to the rapidly clinical evolution, abdominal computed tomography (CT) was performed and showed a frank distension of the gallbladder with thickening of the walls and important densification of the vascular bed. Also coexisted a radiopaque calculation jammed in the infundibular region. Free air within the gallbladder wall was not seen. No intra-hepatic or extra-hepatic duct dilatation was seen (Figure 1(a) and Figure 1(b)). From leukocytosis, history of diabetes and CT findings, we diagnosed the patient as having acute bacterial calculous cholecystitis and she was referred to the department of surgery. The patient started a conservative therapy with intravenous piperacillin and tazobac$\operatorname{tam}$ (4.5 g thrice daily) for 9 days with clinical and analytical improvement (C-reactive protein $6.5 \mathrm{mg} / \mathrm{dL}$; RR, 
$<0.5 \mathrm{mg} / \mathrm{dL}$ without leukocytosis). After that period, the patient was clinically asymptomatic and was submitted to a laparoscopic cholecystectomy under general anaesthesia uneventfully. The gallbladder was found to be gangrenous. Pathologic examination revealed findings suggestive of gangrenous cholecystitis and hemorrhagic changes, mural necrosis and suppurative inflammation (Figure 2(a) and Figure 2(b)). No gallbladder stones were found in the specimen. Postoperatively the patient recovered well and she was discharged from the hospital on the third postoperative day.

\section{Discussion}

In elderly patients, numerous factors subsidize the diagnostic challenge and high incidence of complications in this specific population. Immune function decreases with advancing age and most of them have underlying con-

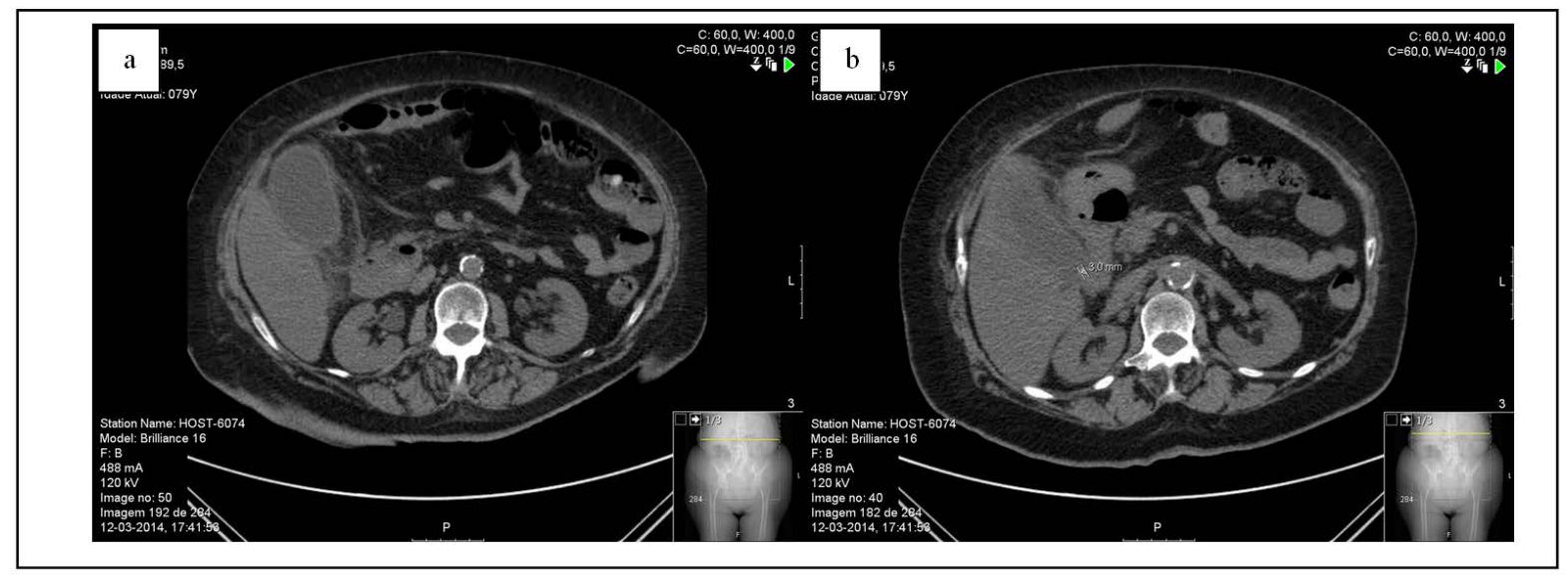

Figure 1. (a) and (b) Abdominal enhanced computed tomography showed enlarged gallblader, thicknening of the wall and a radiopaque calculation jammed in the infundibular region.

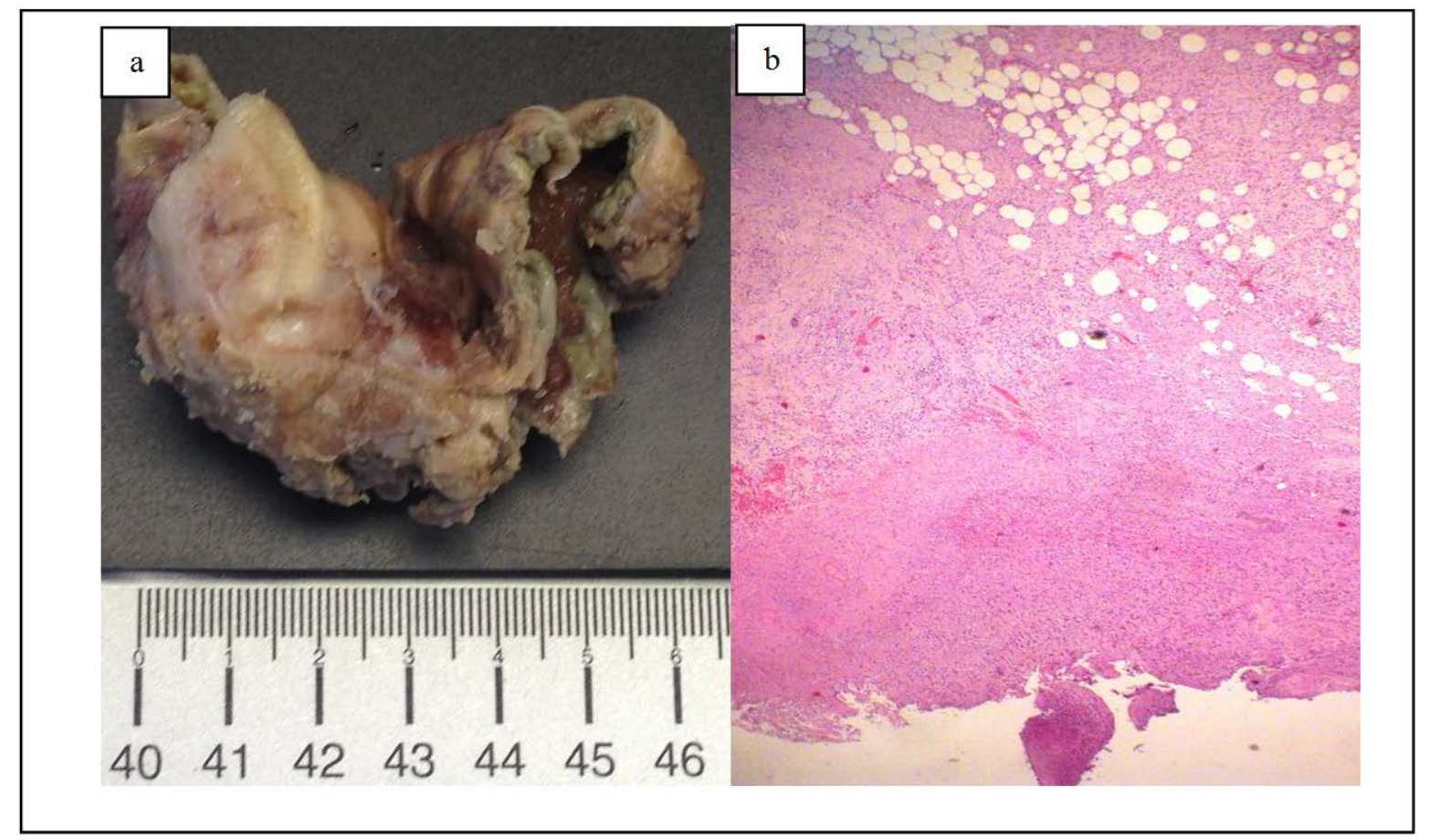

Figure 2. (a) and (b) Pathologic examination of the gallblader revealed a gangrenous cholecystitis: macrocopically with scale marker in centimeters and microscopically with hematoxylin and eosin stain, amplified 400×. 
ditions as diabetes mellitus, concomitantly suppressing immunity. Underlying cardiovascular and pulmonary disease also plays an important role decreasing physiologic reserve. Additionally, they tend to wait longer to seek medical attention, are more likely to present with vague symptoms and nonspecific findings on presentation and there is a higher incidence of asymptomatic underlying pathology. Diminished sensorium is common and permits pathology to advance to a risky point prior to symptom development [9]. Due to the described factors, many elderly patients are misdiagnosed with relatively benign conditions and are at greater risk of being admitted to the wrong service (i.e., internal medicine when a surgeon may be required as realized in our case). A cautious history, physical examination and a high index of suspicion are critical to prevent missed diagnoses. Acute cholecystitis is a critical example of how the previous factors are likely to play an important role in the progression towards complications as gangrenous cholecystitis. Gangrenous cholecystitis is considered a more severe form of acute cholecystitis. The incidence of gangrenous cholecystitis ranges from $2 \%$ to $29.6 \%$ in all patients with acute cholecystitis [4] and constitutes is a medical and surgical emergency.

Acute cholecystitis may present in a different way in special populations, especially elderly diabetics. It may present with indefinite symptoms and without many historical and physical findings, with localized tenderness as the only presenting sign and may progress to complicated cholecystitis rapidly and without warning. In general populations, the most common presenting symptom of acute cholecystitis is upper abdominal pain. Commonly, the pain starts in the epigastric region and then localizes to the right upper quadrant. Signs of peritoneal irritation may be present. The physical examination may disclose fever, tachycardia, and tenderness in the epigastric region that localizes to the right upper quadrant (RUQ), often with guarding or rebound. However, the absence of physical findings does not rule out the diagnosis of cholecystitis. Therefore, diagnosing elderly diabetics as having acute cholecystitis, even when complicated, may be a clinical challenge. Abdominal pain poses a difficult challenge in elderly people, may be the only symptom in a wide range of diseases and their pain is likely to be much less severe than expected for a particular disease. Additionally, they are less likely to have fever, leukocytosis, or elevated C-reactive protein level.

The diagnosis may comprise laboratory criteria, nevertheless these are not consistent in recognizing all patients with the pathology, as in our patient, since laboratory testing with exception of leukocytosis and gammaglutamyl transpeptidase, were within the normal range during the entire evolution of the disease. Laboratory criteria may reveal several abnormal findings as leukocytosis with a left shift; alanine aminotransferase (ALT) and aspartate aminotransferase (AST) levels may be raised in cholecystitis, with bile duct obstruction and assess the occurrence of hepatitis; bilirubin and alkaline phosphatase levels also evaluate evidence of duct obstruction and may be elevated in up to $25 \%$ of patients; amylase/lipase assays evaluate the presence of pancreatitis; mildly elevation of amylase may present in cholecystitis. Urinalysis is used to rule out pyelonephritis and renal calculi.

Ultrasonography is the desired initial imaging test for the diagnosis of acute cholecystitis, though the precision of this preoperative exam in identifying gangrenous cholecystitis remains undefined. However, CT plays as important role in evaluation of these patients if ultrasonography is inconclusive and can identify extrabiliary disorders and complications of acute cholecystitis, such as gangrene, gas formation, and perforation [10]. Presence of wall or lumen gas, irregular wall, lack of mural enhancement, intra-luminal membranes and pericholecystic fluid are findings suggestive of this condition. A superior degree of wall thickening and gallbladder distension may be the only result and is not specific [4]. Several studies have reported some differences concerning GC and NGAC, despite they are not consistent. Patients with GC are more likely to be older, diabetic, male and to present fever, elevated pulse and muscle rigidity on physical examination [11]. Concerning laboratory criteria, leukocytosis, C-reactive protein, bilirubin, urea and creatinine may be greatly increased in GC when compared do NGAC. Our patient prior to surgery, was asymptomatic and her laboratory investigation were normal, which is not consistent with what is currently reported in literature.

Treatment of cholecystitis is determined by the severity of the condition and possible complications. Antibiotics are useful to manage the infection and definitive therapy involves placement of a drainage device or cholecystectomy. Several studies repot high rate of failure with medical management in GC, with early cholecystectomy supported by several studies [12]-[14]. GC has a mortality rate of up to $22 \%$ and a complication rate of $16 \%-25 \%$, including perforation, abscess formation or peritonitis [3].

\section{Conclusion}

Delays in diagnosing acute cholecystitis result in a higher prevalence of morbidity and mortality due to severe 
complications as gangrenous cholecystitis. Pre-operative diagnosis of this condition may be challenging to define. Consequently, the diagnosis should be measured and investigated promptly in order to prevent poor outcomes with particular attention to elderly diabetics.

\section{References}

[1] Contini, S., Corradi, D., Busi, N., Alessandri, L., Pezzarossa, A. and Scarpignato, C. (2004) Can Gangrenous Cholecystitis Be Prevented? A Plea against a “Wait and See” Attitude. Journal of Clinical Gastroenterology, 38, 710-716. http://dx.doi.org/10.1097/01.mcg.0000135898.68155.88

[2] Bingener, J., Stefanidis, D., Richards, M.L., Schwesinger, W.H. and Sirinek, K.R. (2005) Early Conversion for Gangrenous Cholecystitis: Impact on Outcome. Surgical Endoscopy, 19, 1139-1141. http://dx.doi.org/10.1007/s00464-004-8190-9

[3] Grant, R.L. and Tie, M.L.H. (2002) False Negative Biliary Scintigraphy in Gangrenous Cholecystitis. Australasian Radiology, 46, 73-75. http://dx.doi.org/10.1046/j.1440-1673.2001.00998.x

[4] Bennett, G.L., Rusinek, H., Lisi, V., Israel, G.M., Krinsky, G.A., Slywotzky, C.M. and Megibow, A. (2002) CT Findings in Acute Gangrenous Cholecystitis. American Journal of Roentgenology, 178, 275-281. http://dx.doi.org/10.2214/ajr.178.2.1780275

[5] Fry, D.E., Cox, R.A. and Harbrecht, P.J. (1981) Gangrene of the Gallbladder: A Complication of Acute Cholecystitis. Southern Medical Journal, 74, 666-668. http://dx.doi.org/10.1097/00007611-198106000-00007

[6] Fagan, S.P., Awad, S.S., Rahwan, K., Hira, K., Aoki, N., Itani, K.M., et al. (2003) Prognostic Factors for the Development of Gangrenous Cholecystitis. American Journal of Surgery, 186, 481-485. http://dx.doi.org/10.1016/j.amjsurg.2003.08.001

[7] Stefanidis, D., Bingener, J., Richards, M., Schwesinger, W., Dorman, J. and Sirinek, K. (2005) Gangrenous Cholecystitis in the Decade before and after the Introduction of Laparoscopic Cholecystectomy. JSLS, 9, 169-173.

[8] Stefanidis, D., Sirinek, K.R. and Bingener, J. (2006) Gallbladder Perforation: Risk Factors and Outcome. Journal of Surgical Research, 131, 204-208. http://dx.doi.org/10.1016/j.jss.2005.11.580

[9] Laurell, H., Hansson, L.E. and Gunnarsson, U. (2006) Acute Abdominal Pain among Elderly Patients. Gerontology, 52, 339-344. http://dx.doi.org/10.1159/000094982

[10] Katz, D.S., Rosen, M.P., Blake, M.A., et al. (Expert Panel on Gastrointestinal Imaging) (2010) Appropriateness Criteria ${ }^{\circledR}$ Right upper Quadrant Pain. American College of Radiology, Reston.

[11] Nikfarjam, M., Niumsawatt, V., Sethu, A., Fink, M.A., Muralidharan, V., Starkey, G., Jones, R.M. and Christophi, C. (2011) Outcomes of Contemporary Management of Gangrenous and Non-Gangrenous Acute Cholecystitis. International Hepato-Pancreato-Biliary Association, 13, 551-558. http://dx.doi.org/10.1111/j.1477-2574.2011.00327.x

[12] Morrow, D.J., Thompson, J. and Wilson, S.E. (1978) Acutecholecystitis in the Elderly: A Surgical Emergency. Archives of Surgery, 113, 1149-1152. http://dx.doi.org/10.1001/archsurg.1978.01370220035006

[13] Borzellino, G., Sauerland, S., Minicozzi, A.M., Verlato, G., Di Pietrantonj, C., de Manzoni, G., et al. (2008) Laparoscopic Cholecystectomy for Severe Acute Cholecystitis. A Meta-Analysis of Results. Surgical Endoscopy, 22, 8-15. http://dx.doi.org/10.1007/s00464-007-9511-6

[14] Chau, C.H., Siu, W.T., Tang, C.N., Ha, P.Y., Kwok, S.Y., Yau, K.K., et al. (2006) Laparoscopic Cholecystectomy for Acute Cholecystitis: The Evolving Trend in an Institution. Asian Journal of Surgery, 29, 120-124. http://dx.doi.org/10.1016/S1015-9584(09)60069-5 
Scientific Research Publishing (SCIRP) is one of the largest Open Access journal publishers. It is currently publishing more than 200 open access, online, peer-reviewed journals covering a wide range of academic disciplines. SCIRP serves the worldwide academic communities and contributes to the progress and application of science with its publication.

Other selected journals from SCIRP are listed as below. Submit your manuscript to us via either submit@scirp.org or Online Submission Portal.
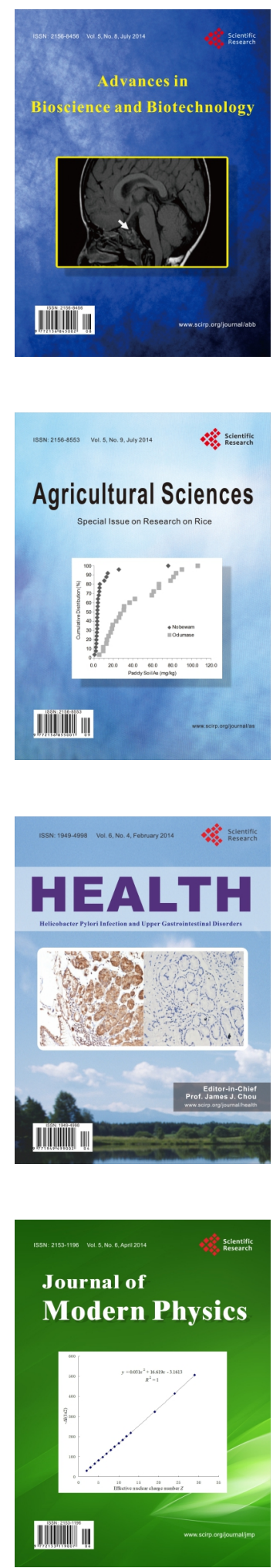
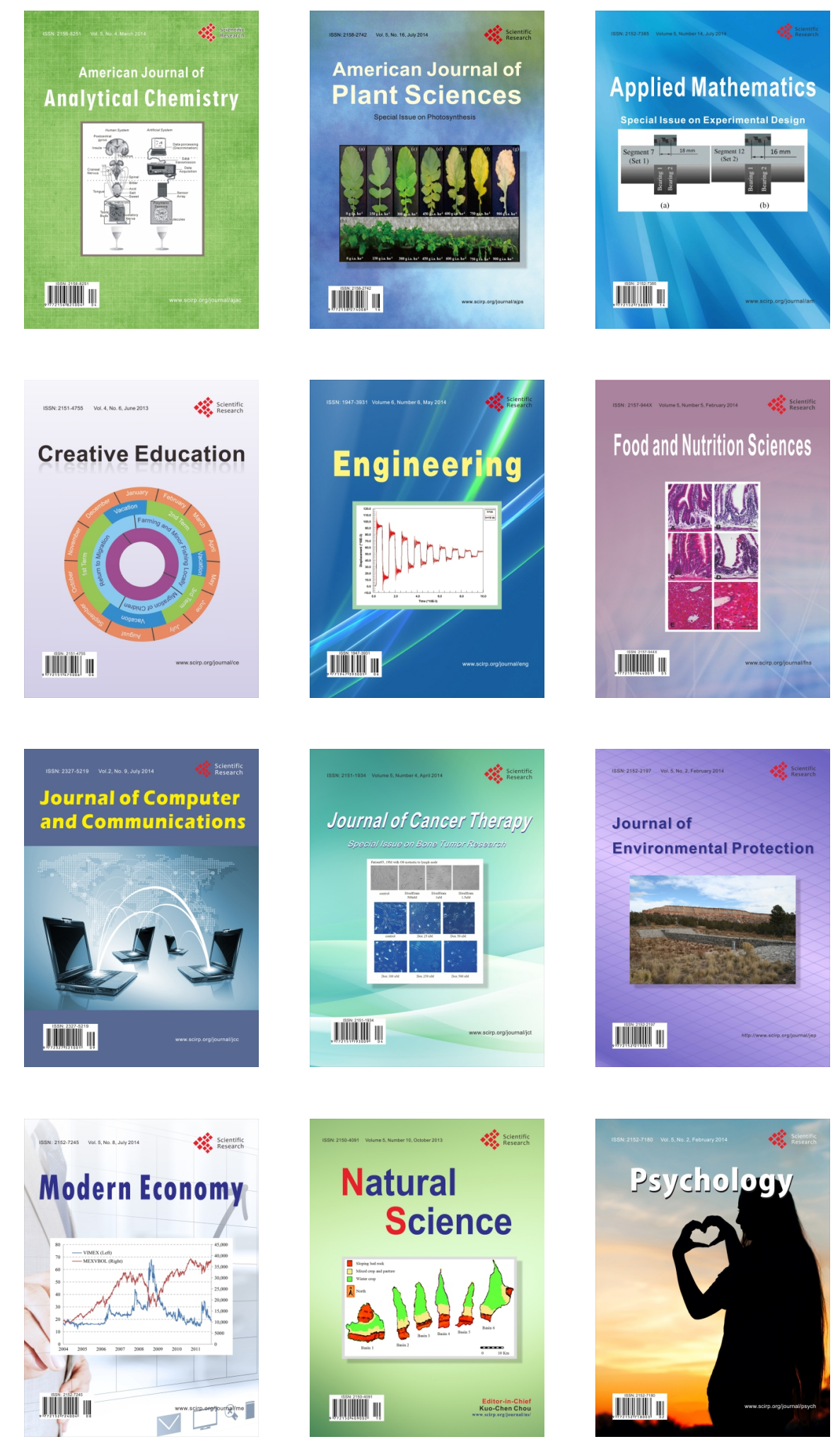